\title{
On the suitability of near-infrared (NIR) systems for next-generation brain-computer interfaces
}

\author{
Shirley Coyle ${ }^{1}$, Tomás Ward ${ }^{1}$, Charles Markham ${ }^{2}$ and Gary McDarby ${ }^{3}$ \\ ${ }^{1}$ Department of Electronic Engineering, National University of Ireland Maynooth, Maynooth, \\ Co. Kildare, Ireland \\ 2 Department of Computer Science, National University of Ireland Maynooth, Maynooth, \\ Co. Kildare, Ireland \\ ${ }^{3}$ Media Lab Europe, Dublin, Ireland
}

Received 5 February 2004, accepted for publication 9 June 2004

Published 22 July 2004

Online at stacks.iop.org/PM/25/815

doi:10.1088/0967-3334/25/4/003

\begin{abstract}
A brain-computer interface (BCI) gives those suffering from neuromuscular impairments a means to interact and communicate with their surrounding environment. A BCI translates physiological signals, typically electrical, detected from the brain to control an output device. A significant problem with current BCIs is the lengthy training periods involved for proficient usage, which can often lead to frustration and anxiety on the part of the user. Ultimately this can lead to abandonment of the device. The primary reason for this is that relatively indirect measures of cognitive function, as can be gleaned from the electroencephalogram (EEG), are harnessed. A more suitable and usable interface would need to measure cognitive function more directly. In order to do this, new measurement modalities, signal acquisition and processing, and translation algorithms need to be addressed. In this paper, we propose a novel approach, using non-invasive near-infrared imaging technology to develop a user-friendly optical BCI. As an alternative to the traditional EEGbased devices, we have used practical non-invasive optical techniques to detect characteristic haemodynamic responses due to motor imagery and consequently created an accessible BCI that is simple to attach and requires little user training.
\end{abstract}

Keywords: brain-computer interface, near-infrared technology, functional brain imaging, motor imagery

(Some figures in this article are in colour only in the electronic version) 


\section{Introduction}

\subsection{Brain-computer interfacing}

Brain-computer interfaces (BCIs) provide users with an alternative output channel other than the normal output path of the brain, i.e. the efferent nervous system and muscles. The purpose of a $\mathrm{BCI}$ is to detect physiological signals from the brain and translate them into a control signal for an external device such as a communication aid or prosthetic limb. Current generation BCIs generally rely on the brain's electrical activity measured using surface EEG but implanted electrodes are also used (Wolpaw et al 2000). Signals used include visual evoked potentials (VEP), slow cortical potentials, P300 evoked potentials, mu and beta rhythms (sensorimotor rhythms) (Wolpaw et al 2002). Some systems rely on physiological signals that are associated with movement that can be controlled using motor imagery (Kübler et al 2001, Pfurtscheller et al 2001). Other systems use the P300 evoked response associated with the 'oddball' paradigm. Physiological signals due to thoughts corresponding to natural intent are preferable. A summary of current BCI research is shown in table 1 (Wolpaw 2002). To operate such a BCI, users must learn to control their brain's electrical signals. Learning techniques to command brain activity that ordinarily may not be consciously controlled are extremely difficult and can take months of training. This can cause a great deal of frustration and sometimes abandonment of the device (Spinney 2003). There is a need for a more accessible interface that uses a more discernible measure of cognitive function in order to control an output device. We propose a novel approach to BCI control signal acquisition, involving the use of near-infrared light, to create a more practical and user-friendly BCI.

\subsection{Near-infrared functional brain imaging}

Near-infrared light can penetrate the human adult head to sufficient depths so as to allow functional mapping of the cerebral cortex. Changes in tissue oxygenation associated with brain activity modulate the absorbtion and scattering of these NIR photons. By measuring the optical changes at various wavelengths in the NIR band qualitative measures of brain activity can be obtained. This is the basis of the near-infrared techniques described in this communication. The NIR response in the brain is comprised of two signals. There is a slow response (approx. 5-8 s) as a result of attenuation changes due to cerebral haemodynamics (yielding localised blood volume and oxygenation changes) and a fast response (of the order of milliseconds) that is thought to be due to changes in the scattering properties of the neuronal membranes during firing (Stepnoski et al 1991). This signal is more directly related to neural activity and may correlate with evoked potentials commonly used in EEG analysis. It has been termed the Event Related Optical Signal (Gratton et al 2001, Wolf et al 2002).

Our current BCI system focuses on the slow vascular response, which can be continuously monitored in real-time. The fast response is of much smaller magnitude and requires averaging techniques to achieve acceptable signal to noise ratios. Franceschini and Boas (2003) reported averaging blocks of 1000 trials and observing a $0.05 \%$ change in light intensity. Since this technique was first reported in 1977 (Jobsis 1977), various studies have examined brain activity due to motor, cognitive, visual and auditory functions (Okada et al 1997, Benaron et al 2000, Wolf et al 2002, Izzetoglu et al 2003, Syré et al 2000, Keenan et al 2002) using this modality.

\subsection{An optical BCI}

Most of the traditional EEG-based BCI are difficult to use because they involve directing brain activity that we normally have no control over (Spinney 2003). Recently, researchers 
Table 1. BCI development using various traits of EEG.

\begin{tabular}{|c|c|c|}
\hline Author & EEG signal & Application/performance \\
\hline Vidal (1977) & Visual evoked potentials & Direction of eye gaze controlling cursor \\
\hline Sutter (1992) & Visual evoked potentials & Word-processing program, $10-12$ bits $/ \mathrm{min}$ \\
\hline Middendorf et al (2000) & Visual evoked potentials & $\begin{array}{l}\text { Selection of one of two virtual buttons, } \\
\text { selection time } 2.1 \mathrm{~s} \text {, accuracy } 92 \%\end{array}$ \\
\hline Donchin et al (2000) & P300 evoked potentials & $\begin{array}{l}\text { Communication aid, } 7.8 \text { characters } / \mathrm{min} \text {, } \\
80 \% \text { accuracy }\end{array}$ \\
\hline Birbaumer et al (2000) & Slow cortical potentials & $\begin{array}{l}\text { Thought translation device (TTD), } \\
\text { communication aid, } 0.5 \text { characters } / \mathrm{min}\end{array}$ \\
\hline Wolpaw et al (2003) & $\begin{array}{l}\text { Sensorimotor cortex } \\
\text { rhythms }\end{array}$ & $\begin{array}{l}\text { BCI } 2000, \text { mu and beta rhythm to control } \\
\text { 1D and } 2 \text { D cursor movement, } \\
>95 \% \text { accuracy, } 20-25 \text { bits } / \mathrm{min}\end{array}$ \\
\hline Pfurtscheller et al (2001) & $\begin{array}{l}\text { Sensorimotor cortex } \\
\text { rhythms }\end{array}$ & $\begin{array}{l}\text { Graz BCI-Hand orthosis, } \\
\text { communication aid } \\
>80 \% \text { accuracy } 17 \text { bits } / \mathrm{min}\end{array}$ \\
\hline Kennedy et al (2000) & $\begin{array}{l}\text { Activity of cortical } \\
\text { neurons, using implanted } \\
\text { electrodes }\end{array}$ & $\begin{array}{l}\text { Communication aid, } 3 \text { letters } / \mathrm{min} \text { after } \\
\text { several months training }\end{array}$ \\
\hline
\end{tabular}

demonstrated the use of implanted multi-electrode arrays in the motor cortex of monkeys enabling instant neural control to overcome this problem (Serruya et al 2002). As an alternative method to this invasive approach, the authors have investigated the feasibility of using a noninvasive optical technique, which is both safe and practical, to record more directly measures of cognitive function in humans, and consequently created an accessible BCI requiring little user training.

\section{Methods}

\subsection{Signal acquisition}

The simplest BCI requires a binary (yes/no) signal, which is achievable using a single-channel system. Optode placement and the protocol used to elicit the yes/no signal are user-dependent issues in terms of maximum signal strength, ease of use and accuracy. Our current NIR system consists of a single channel arrangement operating at two different wavelengths. Various chromophores found in tissue, e.g. oxy-haemoglobin and deoxy-haemoglobin, have different attenuation spectra, which means that multi-wavelength systems can monitor changes in such chromophore concentrations (Villringer and Chance 1997). The light sources are $700 \mathrm{~nm}$ and $880 \mathrm{~nm}$ emitting a combined average power of less than $2 \mathrm{~mW} \mathrm{~mm}^{-2}$. The optical radiation of light emitting diodes (LEDs) differs from laser sources, placing them between incoherent broadband and coherent laser sources. Currently there are no maximum permissible exposure (MPE) for skin values specified for LEDs (Horak 1999). Nevertheless, the MPE of skin for a laser at $880 \mathrm{~nm}$, for an exposure time up to 8 hours, is $4.6 \mathrm{~mW} \mathrm{~mm}^{-2}$. The LEDs are placed in direct contact with the scalp, and modulated at $4 \mathrm{kHz}$ and $5 \mathrm{kHz}$ respectively to enable lock-in detection at each wavelength. The detector is an avalanche photodiode (APD) (Hamamatsu C5460-01) that is connected 3-4 cm from the light source via a $3 \mathrm{~mm}$ diameter fibre optic bundle. Two lock-in amplifiers (Ametek 5210) are used to obtain the intensities of the two wavelengths of light and reduce noise due to ambient light. The data is recorded at a sampling 


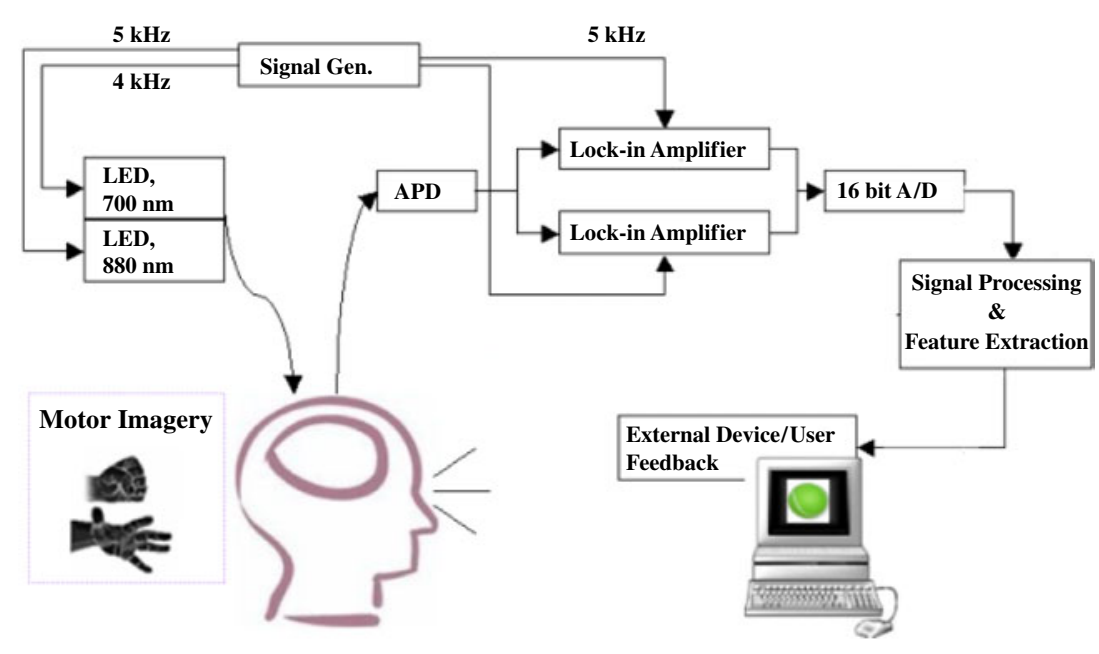

Figure 1. Optical BMI architecture.

rate of $100 \mathrm{~Hz}$ by a 16-bit A/D data acquisition card (Keithley PCMCIA16AI). The system architecture is shown in figure 1.

\subsection{Mental tasks for BCI control}

The mental task involved must be straightforward and relate to natural intent in order to make the device user-friendly. Motor imagery, i.e. the imagination of physical movement, is one possibility for this protocol. Motor imagery is known to activate similar cortical areas as real executed movements. Other researchers have demonstrated optical responses resulting from the performance of motor tasks and shown a peak response occurring at 5-8 s after the onset of movement (Benaron et al 2000). The authors have recently measured an optical response due to motor imagery at $\mathrm{C} 3 / \mathrm{C} 4$ of the 10-20 electrode placement system. In that work motor imagery was found to give a similar optical response to actual executed movement (Coyle et al 2003). Analysis of the signals offline showed characteristic changes in the haemoglobin concentrations during mental activation. Our current BCI is based on these findings - such characteristics of the vascular response due to motor imagery, that differ from the response during rest intervals, can be detected in real-time and subsequently translated to a control output.

\subsection{Signal analysis and feature extraction}

The optical response is derived from two dynamic processes. A slow response occurs after 5-8 s resulting from absorption and scattering changes due to the cerebral vascular response. Cortical activity leads to haemodynamic changes associated with the increase in metabolism. Our current NIR system targets the slow vascular response, as its larger signal magnitude is at present a more accessible option for BCI development. The system performance is limited by the nature of the physiological signal. Our initial trials identified significant changes in oxy-haemoglobin levels using a straightforward algorithm. The average oxy-haemoglobin concentration level is calculated every second and a $20 \mathrm{~s}$ window of data is analysed. An event is noted if the average oxy-haemoglobin concentration is greater than a reference level. The reference is set to the maximum level occurring during the first ten seconds of the window. This is done to take into account spontaneous low frequency oscillations that are inherent in 


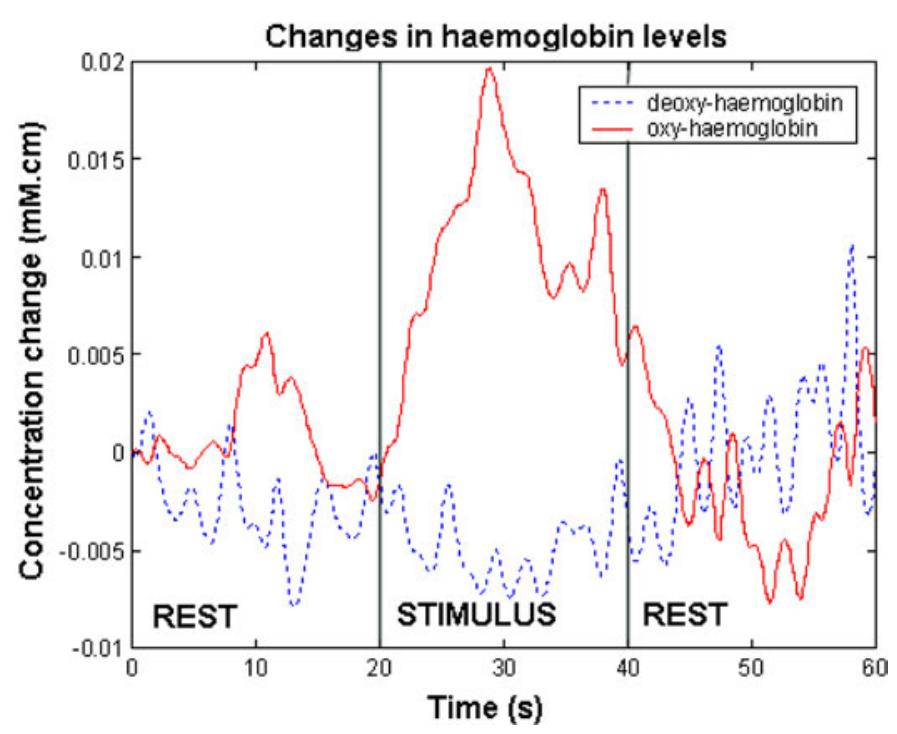

Figure 2. Characteristic haemodynamic response due to motor imagery.

the signal. Such oscillations are thought to be due to blood pressure fluctuations and usually have a period of 10-15 s (Elwell et al 1999). Identification of an event is registered by the $\mathrm{BCI}$ and feedback is given to the user.

\subsection{Experimental procedure}

Subjects possessing full motor control participated in this study. The optical response was measured using a single channel on the surface of the scalp above the motor cortex. The optode was placed at position C3, according to the EEG 10-20 system, an area known to be associated with hand movement. The subjects were asked to clench a rubber ball or to visualise doing so using the hand contralateral to the side of optode placement. Such mental tasks lead to a localised increase in cerebral blood flow supplying oxygen in response to the increased metabolic rate. Oxygen supply exceeds demand, consequently causing an increase in oxy-haemoglobin and decrease in deoxy-haemoglobin concentrations (Villringer and Dirnagl 1995). Identification of such an event is registered by the BCI and feedback, an essential aspect of all BCIs, is given to the user. For motor imagery tasks, the optical BCI delivers continuous visual feedback, as the user imagines clenching a ball, by means of a circle on the screen that shrinks and expands in response to changing haemoglobin levels. The purpose of using visual feedback is to reinforce the mental imagery being used.

\section{Results}

\subsection{Optical response}

Hand movement and imagined hand movement initiate neural activity within the motor cortex of the contra-lateral hemisphere (Beisteiner et al 1995). A few seconds after the onset of movement, due to a slow vascular response, an increase in oxy-haemoglobin occurs and a decrease in deoxy-haemoglobin. On completion of the task the concentration levels return to baseline values. The typical optical response due to a motor imagery task, recorded at position $\mathrm{C} 3$, for a single trial is shown in figure 2. This signal has been linearly detrended 
and low-pass filtered using a fourth order Butterworth filter with a cut-off frequency of $0.5 \mathrm{~Hz}$, before applying the modified Beer-Lambert law to calculate haemoglobin concentration changes (Wyatt et al 1990).

\subsection{BCI performance}

Our current system based on the slow vascular response uses a single optode and a very basic signal processing methodology to identify the signal transition between rest and mental activation. On account of the $20 \mathrm{~s}$ window required for this basic translation algorithm, the system has an information transfer rate currently of 3 bits $\mathrm{min}^{-1}$. Single channel systems with improved signal processing have potential data rates of 6 bits $\mathrm{min}^{-1}$, assuming the allowance of approximately $5 \mathrm{~s}$ for the response to register and $5 \mathrm{~s}$ for the signal to return to a baseline state. Higher information transfer rates using this slow response are possible by using multiple channels.

Analysis of offline data averaged over a number of subjects and trials by applying the BCI's translation algorithm gave an accuracy of $75 \%$. Further development of signal processing techniques to remove the effects of physiological noise and motion artefacts will improve these rates e.g. adaptive filtering techniques will allow easier separation of the response from unrelated physiological activity.

The great advantage of this system is the fact that training is so simple because the mental task involved does not require the user to master a new thought process. Subjects are asked to carry out a motor task initially, typically clenching a tennis ball, and then asked to visualise the task. This is a natural thought process resulting in naïve users achieving immediate proficiency.

\section{Discussion}

This study has demonstrated the feasibility of an optical BCI controlled by motor cortex activity. Future developments need not be confined solely to motor functions. Functional NIR imaging studies have revealed optical responses resulting from cognitive, visual and auditory functions (Benaron et al 2000, Izzetoglu et al 2003, Syré et al 2000, Keenan et al 2002). Problem solving tasks activating the frontal lobes, concentration and memory related tasks in particular, have been shown to elicit NIR responses (Benaron et al 2000, Izzetoglu et al 2003). It is the opinion of the authors of this paper that such optical responses could also be harnessed for BCI applications. Such signals are relatively easy for a user to control as they are more directly coupled to the cognitive activity involved. Habituation may be a problem with this approach, however as yet no studies have confirmed such an effect. Another option is to use event related optical responses that have been shown to correlate with the EEG evoked responses (Syré et al 2000, Keenan et al 2002). Event-related auditory oddball experiments, which produce the conventional EEG P300 response, result in optical responses consistent with fMRI studies (Keenan et al 2002). In some current EEG-based BCIs, the P300 signal is used. Although the time-course of the signal is much smaller, $300 \mathrm{~ms}$, compared to the optical signal, $5.8 \pm 0.3 \mathrm{~s}$ (Keenan et al 2002), the latter technique requires considerable averaging (Vaughan 2003).

Higher data rates can be achieved by increasing the number of channels in the system, i.e. increasing the number of bits per baud. Integration of an additional optode above the opposite motor cortex will be one of the next steps in order to offer horizontal cursor control. Another method to increase the throughput is to increase the baud rate. To do this, the fast optical response must be sought, a signal which researchers have only recently begun to pursue. 


\section{Conclusions}

Speed, accuracy, ease of use and length of training period are the key criteria for BCI development. Currently no BCI performs well in all these aspects (Wolpaw et al 2000). Greater accuracy rates and speed are needed particularly for neuroprosthesis control. Ease of use and training periods must be addressed in order for BCIs to be successful in terms of accessibility. Many of the current BCIs require the user to learn a new thought process, which requires immense effort and can lead to frustration. In order to further BCI development, signal acquisition, signal processing and translation algorithms need to be addressed. This paper demonstrates the suitability of NIR to detect changes in physiological signals, detected non-invasively, in order to control a BCI. The thought processes required are relatively straightforward and are a more direct measure of cognitive function. While our current system is limited by the nature of the slow vascular response, recent studies have revealed a fast optical signal (Gratton and Fabiani 2001, Wolf et al 2002), showing that there is continuing development of NIR imaging technology.

Trials to date have been carried out with able-bodied subjects. Future validation of the system must involve trials with paralysed subjects in order to investigate attributes of motor cortical function. Previous studies have shown motor cortex activation in severely paralysed subjects resulting from motor imagery tasks (Mao et al 1998, Shoham et al 2001). Clinical trials would be needed to examine the effects of fatigue and administered drugs on functional activation of the cerebral cortex and associated haemodynamic changes. BCIs utilizing the electrical signals from the brain have shown reduced performance due to fatigue, toxicity from infections, pain and analgesics (Kennedy 2000).

At present electroencephalography (EEG) using either surface or implanted electrodes, is the most favored technique for BCI development (Wolpaw et al 2002). An optical approach is not susceptible to mains interference or disturbances from other electrical equipment, which affects EEG signals. Optical techniques have other various safety benefits-the user is electrically isolated from the hardware, NIR light is non-ionizing and is therefore suitable for long-term use, and the technique takes a completely non-invasive approach. Other functional imaging methods such as functional magnetic resonance imaging (fMRI) and magnetoencephalography (MEG) have been used for BCI development but are at present impractical. Both MEG and fMRI systems are large, need a magnetically shielded environment and are very expensive. In addition, NIR instrumentation is straightforward to use and does not require the use of gel to make electrical contact with the skin. As with near-infrared spectroscopy (NIRS) bedside-monitoring apparatus, NIR techniques offer the promise of a non-invasive brain-computer interface system that is portable, cost-effective, practical and safe for long-term use.

\section{References}

Beisteiner R, Höllinger P, Lindinger G, Lang W and Berthoz A 1995 Mental representations of movements. Brain potentials associated with imagination of hand movements Electroencephalogr. Clin. Neurophysiol.96 183-93

Benaron D et al 2000 Noninvasive functional imaging of human brain using light J. Cerebral Blood Flow Metabolism 20 469-77

Birbaumer N, Kübler A, Ghanayim N, Hinterberger T, Perelmouter J, Kaiser J, Iversen I, Kochoubey B, Neumann N and Flor H 2000 The thought translation device (TTD) for completely paralyzed patients IEEE Trans. Rehab. Eng. 8 190-2

Coyle S, Ward T, Markham C and McDarby G 2003 Cerebral blood flow changes related to motor imagery using near-infrared spectroscopy (NIRS) World Cong. Med. Phys. Biomed. Eng. (Sydney, Australia) IFMBE Proceedings 4 
Donchin E, Spencer K M and Wijesinghe R 2000 The mental prosthesis: assessing the speed of a P300-based brain-computer interface IEEE Trans. Rehab. Eng. 8 174-9

Elwell C E, Springett R, Hillman E and Delpy D T 1999 Oscillations in cerebral haemodynamics Oxygen Transport to Tissue XXI ed A Eke and D T Delpy (New York: Kluwer Academic/Plenum) pp 57-65

Franceschini M A and Boas D A 2003 Non-invasive fast optical measurement of neuronal activity Proc. Euro. Conf. Biomedic. Opt. (Munich, Germany) 5138-52

Gratton G and Fabiani M 2001 Shedding light on brain function: the event-related optical signal Trends Cognitive Science 5 357-63

Horak W 1999 Risk assessment of light emitting diodes J. Laser Appl. 11 21-6

Izzetoglu K, Yurtsever G, Bozkurt A, Yazici B, Bunce S, Pourrezaei K and Onaral B 2003 NIR spectroscopy measurements of cognitive load elicited by GKT and target categorization Proc. 36th Hawaii Int. Conf. Syst. Sci. (HICSS'03)

Jobsis F 1977 Non-invasive infrared monitoring of cerebral and myocardial oxygen sufficiency and circulatory parameters Science 198 1264-7

Keenan R, Horovitz S, Maki A, Yamahita Y, Koizumi H and Gore J 2002 Simultaneous recording of event-related auditory oddball response using transcranial near infrared optical topography and surface EEG Neuroimage 16 587-92

Kennedy P R, Bakay R A E, Moore M M, Adams K and Goldwaithe J 2000 Direct control of a computer from the human central nervous system IEEE Trans. Rehabil. Eng. 8 198-202

Kübler A, Kotchoubey B, Kaiser J, Wolpaw J and Birbaumer N 2001 Brain-computer communication: unlocking the locked in Psychol. Bull. 127 358-75

Mao H, Muthupillai R, Kennedy P R, Popp C A and Song A W 1998 Clinical application of fMRI: Motor cortex activation in a paralysed patient Medicamundi 43 19-22

Middendorf M, McMillan G, Calhoun G and Jones K S 2000 Brain-computer interfaces based on steady-state visual evoked response IEEE Trans. Rehabil. Eng. 8 211-3

Okada E, Firbank M, Schweiger M, Arridge S, Cope M and Delpy D 1997 Theoretical and experimental investigation of the near-infrared light propagation in a model of the adult head Appl. Opt. 36 21-31

Pfurtscheller G and Neuper C 2001 Motor imagery and direct brain-computer communication Proc. IEEE 89 1123-34

Serruya M D, Hatsopoulos N G, Paninski L, Fellows M R and Donoghue J P 2002 Instant neural control of a movement signal Nature 416 141-2

Shoham S, Halgren E, Maynard E M and Normann R A 2001 Motor-cortical activity in tetraplegics Nature 413793

Spinney L 2003 Hear my voice New Scientist 22 36-9

Stepnoski R A, LaPorta A, Raccuia-Behling F, Blonder G E, Slusher R E and Kleinfeld D 1991 Noninvasive detection of changes in membrane potential in cultured neurons by light scattering Proc. Natl Acad. Sci. USA $889382-6$

Sutter E E 1992 The brain response interface: communication through visually induced electrical brain responses J. Microcomput. Appl. 15 31-45

Syré F, Obrig H, Steinbrink J, Kohl M, Wenzel R and Villringer A 2000 Are VEP correlated fast optical changes detectable in the adult by non-invasive near infrared spectroscopy (NIRS)? Adv. Exp. Med. Biol. 471

Vaughan T M 2003 Brain-computer interface technology: a review of the second international meeting IEEE Trans. Neural Syst. Rehabil. Eng. 11 94-109

Vidal J J 1977 Real-time detection of brain events in EEG IEEE Proc. Special Issue on Biological Signal Processing and Analysis $\mathbf{6 5} 633-64$

Villringer A and Chance B 1997 Non-invasive optical spectroscopy and imaging of human brain function Trends Neurosci. 20 435-42

Villringer A and Dirnagl U 1995 Coupling of brain activity and cerebral blood flow: basis of functional neuroimaging Cerebrovasc. Brain Metabolism Rev. 7 240-76

Wolf M et al 2003 Near-infrared spectroscopy detects fast functional neuronal signals of the visual and motor cortex World Cong. Med. Phys. Biomed. Eng. (Sydney, Australia) IFMBE Proc. 4

Wolf M, Wolf U, Choi J, Gupta R, Safonova L, Paunescu L, Michalos A and Gratton E 2002 Functional frequencydomain near-infrared spectroscopy detects fast neuronal signal in the motor cortex Neuroimage 17 1868-75

Wolpaw J et al 2000 Brain-computer interface technology: a review of the first international meeting IEEE Trans. Rehabil. Eng. 8 164-73

Wolpaw J, Birbaumer N, McFarland D, Pfurtscheller G and Vaughan T 2002 Brain-computer interfaces for communication and control Clin. Neurophysiol. 113 767-91

Wolpaw J R, McFarland D J, Vaughan T M and Schalk G 2003 The Wadsworth Center brain-computer interface (BCI) research and development program IEEE Trans. Neural Syst. Rehabil. Eng. 11 204-7

Wyatt J, Cope M, Delpy D T, Richardson C E, Edwards A D, Wray S and Reynolds E O 1990 Quantitation of cerebral blood volume in human infants by near-infrared spectroscopy J. Appl. Physiol. 68 1086-91 